\title{
Situación Actual de los Programas de Evaluación Académica de la Educación Superior Mexicana: Sus Efectos en el Trabajo Académico
}

\author{
Current Situation of Academic Evaluation Programs in Mexican \\ Higher Education: Its Effects on Academic Work
}

\author{
Patricia Covarrubias-Papahiu * \\ Facultad de Estudios Superiores Iztacala-UNAM
}

\begin{abstract}
A casi tres décadas que entraron en vigor las políticas de evaluación académica, derivadas de las políticas públicas del gobierno mexicano, cuyo propósito fue elevar la calidad educativa de la educación superior, este ensayo tiene como propósito analizar su condición actual, recuperar sus aciertos, desaciertos y paradojas que impactan las condiciones laborales y prácticas de sus académicos. Para ello se hace un recuento de los programas de evaluación e incentivos a la productividad académica, y se recuperan los hallazgos de varias investigaciones que indagan, desde la propia perspectiva de los actores, su situación actual para discutirlos a la luz del debate que sobre el tema se ha publicado. Se concluye que aún quedan pendientes acciones institucionales que vinculen la evaluación académica con el desarrollo profesional de los docentes y el mejoramiento en la calidad de la educación superior, en tanto surgen otros temas emergentes que hoy requieren su atención
\end{abstract}

Palabras claves: Políticas gubernamentales, Políticas de la educación, Educación superior pública, Evaluación docente, Programas de evaluación, Trabajo docente, Desempeño docente, Incentivos.

\begin{abstract}
Almost three decades that the academic evaluation policies, derived from the Mexican government's public policies, whose purpose was to raise the quality of higher education, came into force, this essay aims to analyze its current condition, recover its successes, mistakes and paradoxes that impact the working and practical conditions of its academics. In order to do so, the evaluation programs and incentives to academic productivity are recounted and the findings of several investigations are retrieved from the perspective of the actors themselves, in order to discuss them in the light of the debate about the Topic has been published. It is concluded that there are still pending institutional actions that link academic evaluation with the professional development of teachers and improvement in the quality of higher education, while other emerging issues arise that today require their attention
\end{abstract}

Keywords: Government policies, Politics of education, Public higher education, Teacher evaluation, Programs evaluation, Teacher work, Teacher performance, Incentives. 


\section{Introducción ${ }^{1}$}

El desarrollo de la profesión académica en México ha experimentado en las cuatro últimas décadas un crecimiento importante en el número de sus miembros, en la complejidad de las tareas que realizan, y en la importancia que tiene dentro del sistema educativo de nuestro país, como puede constatarse en las tres ediciones de los Estados del Conocimiento de la Investigación Educativa en México (Ducoing y Landesmann, 1996; García, Grediaga y Landesmann, 2003; Saucedo, Guzmán, Sandoval y Galaz, 2013). La investigación que se ha realizado en este campo revela el desarrollo histórico de la profesión académica, de los cambios en las trayectorias profesionales de los académicos, de las características y condiciones de sus espacios de trabajo, de sus experiencias y otras dimensiones más, que permiten conocer más de cerca a uno de los sujetos centrales de la educación en México, en tanto de ellos depende en gran medida el desarrollo de las funciones sustantivas de docencia, investigación, difusión, extensión y gestión universitarias.

Por ello, la importancia que la evaluación del trabajo académico ha cobrado en la agenda de la investigación educativa en México es porque tiene que ver con los diferentes roles del académico, sus condiciones de trabajo, sus redes de relaciones colegiadas, su ingreso económico y condiciones de vida; todas circunscritas en la dinámica de las instituciones de educación superior mexicanas.

Desde los estudios pioneros sobre los académicos en México (Díaz Barriga, 1997; García, 1998; Landesmann, García y Gil, 1996; Rueda, 1998) que analizaron las políticas y procesos de evaluación de los académicos y cómo habían repercutido en sus actividades desde principios de los noventa que entraron en vigor, el campo de estudio sobre los académicos se ha multiplicado y diversificado, por lo que existe una literatura abundante al respecto.

Los estudiosos en este campo advierten cómo las políticas públicas hacia los académicos han tenido consecuencias inesperadas y repercutido negativamente en su desempeño, particularmente en lo que respecta a su compromiso con las instituciones en las que trabajan (Aboites, 1999; García, 1998). En opinión de varios autores, los cambios en el sistema de reconocimiento y recompensas del trabajo académico -que hoy hace excesivo énfasis en la transformación de los insumos y privilegia la dimensión de los resultados en productos de las actividades de investigación-, provocaron un deterioro en el compromiso institucional y social de las actividades que desarrollan los académicos (De Vries, 1998; Ibarra, 2000, citado por Grediaga, 2006).

A casi tres décadas que entraron en vigor las políticas de evaluación académica, y de sostener que poco se ha realizado para solventar los efectos no deseados que los programas e incentivos a la productividad académica promovieron, el propósito de este ensayo es recuperar investigaciones conducidas en este campo que indagan, desde la propia perspectiva de los actores de instituciones de educación superior, cómo han

\footnotetext{
${ }^{1}$ Este trabajo forma parte de la línea de investigación "Procesos curriculares y prácticas pedagógicas en eduación superior", del proyecto de investigación Evaluación y Planeación Educativa, de la Facultad de Estudios Superiores Iztacala, UNAM.
} 
impactado estos programas en sus condiciones de trabajo, su práctica docente y actividades académicas, con la finalidad de tener un acercamiento a la realidad actual.

Antes se hará una revisión de los programas de evaluación e incentivos al desempeño académico más importantes de las instituciones de educación superior públicas a nivel nacional, su origen, objetivos, aplicaciones y condición actual, que permita poner en contexto las investigaciones recuperadas en este trabajo.

\section{Programas de evaluación e incentivos al trabajo académico: sus antecedentes y condición actual}

El tema de la evaluación en la agenda de las políticas educativas en México tiene sus orígenes en el marco internacional con políticas generales impulsadas por organismos internacionales como el Banco Mundial y la UNESCO (Díaz Barriga, 1997), y en México se conjuntaron con procesos locales como el de fuertes recortes presupuestales hacia las universidades públicas en la década de los ochenta, en el entorno de la crisis de la deuda pública; y con la masificación de la enseñanza y diferenciación y diversificación de los centros de enseñanza que derivó en un deterioro de la calidad de la educación superior (Kent, 1986).

En este contexto, la evaluación en la educación superior mexicana se convierte en el centro de las políticas educativas de este nivel, que de acuerdo a Kent (1992/1993) la implementación de estas políticas en relación al desempeño académico incurrió en tres cuestiones de fondo: a) los ingresos del profesorado decrecieron un $40 \%$ después de 1983 , b) no existía la cultura de la evaluación, y c) no había una idea clara de lo que implicaba el trabajo académico, y menos aún cómo evaluarlo. No obstante, el ejercicio de la evaluación académica se vincula a mecanismos de control gubernamental, a procedimientos de estímulo selectivo y a la obtención de incentivos económicos de acuerdo a resultados. La evaluación de rendimientos y productividad en las Instituciones de Educación Superior (IES) se convierte en el componente esencial de la gestión académica (Rodríguez-Gómez, 1998).

Aunque las primeras acciones en materia de evaluación de la educación superior en México datan de los años setenta, en los que se crea el Sistema Nacional de Planeación Permanente de la Educación Superior (SINAPPES) en 1979, derivado de las primeras políticas nacionales para elevar la calidad de las funciones de las IES, y fueron producto de programas de gobierno y de iniciativas de la Asociación Nacional de Universidades e Instituciones de Educación Superior (ANUIES); la evaluación como política se institucionaliza con la publicación del Programa Nacional para la Modernización Educativa (PME) 1989-1994 del gobierno de Salinas de Gortari, que señalaba como prioritario la evaluación permanente, interna y externa de las instituciones para impulsar la mejora de la calidad de los programas educativos y de servicios que se ofrecían, y como meta la creación de una instancia que concibiera un proceso nacional de evaluación de la educación superior (Buendía, 2013). Se crea así la Comisión Nacional de Evaluación de la Educación Superior (CONAEVA) en 1989, que tendría a su cargo dos acciones principales en materia de evaluación: impulsar un proceso nacional de evaluación del sistema con carácter diagnóstico, para determinar el nivel de rendimiento, productividad, eficiencia y calidad, y en lo referente a la evaluación del desempeño 
académico individual, implementaría un programa de becas para lo cual diseñaría diferentes modalidades y organismos para su regulación (Canales, 2008).

Si bien los sistemas de evaluación de las IES entraron en vigor con el PME, en lo que atañe a la evaluación de su personal académico, su discusión antecedió a éste pues ya se contaba con el Sistema Nacional de Investigadores (SNI), como el primer sistema de evaluación al trabajo académico establecido en 1984 (Ordorika y Navarro, 2006).

El SNI se crea como un mecanismo gubernamental en respuesta a la crisis económica de 1982 y destinado a atender la investigación del país mediante el reconocimiento y apoyo directo a los investigadores en activo dedicados a producir conocimiento científico y tecnológico, como una estrategia para evitar la desintegración de su reducida comunidad científica y con ello "detener la fuga de cerebros de las instituciones públicas de educación superior" (Galaz, 2003, p. 84) o para "salvaguardar la planta de académicos dedicada a las labores de investigación que comenzaba a abandonar los recintos universitarios" (Rueda y Torquemada, 2004, p. 30). Desde su inicio estuvo abierto a la investigación en cualquiera de sus ramas y especialidades, y aunque originalmente estuvo limitado a los investigadores de las instituciones del sector público, después se abrió a los investigadores del sector privado (Malo y Rojo, 1996). El reconocimiento se otorga a través de la evaluación por pares y consiste en otorgar el nombramiento de Investigador Nacional (en cualquiera de sus tres niveles), distinción que simboliza la calidad y prestigio de las contribuciones científicas, y en paralelo al nombramiento, se otorgan estímulos económicos cuyo monto varía con el nivel asignado (CONACYT, 2017).

Dentro del PME, la primera iniciativa a nivel nacional dirigida a la evaluación del personal académico de las instituciones públicas de educación superior, es el Programa Nacional de Becas al Desempeño Académico (PBD), establecido en 1990 por la Secretaría de Educación Pública (SEP). Su finalidad fue responder a la problemática salarial que generaba la incertidumbre de permanecer en éstas y compensar y reconocer salarialmente el trabajo académico de aquellos que fuesen más productivos (Díaz Barriga, 1996). Pretendía promover el cambio "cualitativo" a partir del establecimiento de estructuras de promoción del trabajo académico en docencia e investigación, e instituía los incentivos económicos para los resultados de la promoción (ComasRodríguez y Rivera, 2011).

El Gobierno Federal incorporó al subsidio ordinario de las universidades públicas federales, estatales e institutos tecnológicos federales recursos para aplicarse al programa de Becas al Desempeño. Los recursos se otorgaron a los profesores definitivos de tiempo completo en las categorías de Asociado y Titular (se excluye a los Ayudantes de profesor y a los profesores de Asignatura) con tres años de antigüedad, y son beneficios independientes del sueldo, por lo que no constituyen un ingreso fijo regular ni permanente, y por consiguiente no pueden estar sujetos a negociaciones sindicales (Malo y Rojo, 1996; Rubio, 2006).

$\mathrm{Al}$ implantarse el PBD, cada institución universitaria definió los criterios de operación, los reglamentos de funcionamiento interno, los criterios específicos de evaluación, niveles, montos y mecanismos de entrega de los estímulos, así como sus formas de revisión, ajuste y perfeccionamiento del programa. Sin embargo, la mayoría de las instituciones optó por la evaluación mediante indicadores cuantitativos coincidentes con 
el perfil de investigación similares a los del SNI, es decir, en términos de investigación, docencia y difusión de la cultura, criterios que pocos cumplieron al principio por estar orientada su actividad académica a la docencia (Díaz Barriga, 1996; Malo y Rojo, 1996; Canales, 2008).

Por lo que en 1992 se reorienta el PBD hacia uno de Carrera Docente que pretendía ajustarse a los diversos perfiles, ampliándose así a un mayor número de participantes al otorgar beneficios económicos a quienes se dedicaban de manera habitual a las actividades propias de la función docente (Cetina, 2004). Las instituciones que ya operaban el PBD tuvieron inicialmente la opción de manejar los dos programas o fusionar el programa inicial con el de Carrera Docente (Malo y Rojo, 1996).

Para 1994, ambos programas se fusionaron en lo que hoy se conoce como el Programa de Estímulos al Desempeño del Personal Docente (ESDEPED), que aplica para la educación media superior y superior. Para la educación superior tiene como finalidad "estimular y fortalecer los valores inherentes a la enseñanza, el cual tiene como objetivo fundamental valorar y estimular el desarrollo del personal docente en las categorías de tiempo completo, medio tiempo y asignatura que realicen con calidad, dedicación y permanencia las actividades de docencia, investigación, tutorías y participación en cuerpos colegiados" (SEP, 2015a, citado por Ruiz de la Torre y Rueda, s/f). El programa está vigente y opera bajo los Lineamientos Generales de la Secretaría de Hacienda y Crédito Público (SHCP), y los reglamentos internos de cada institución. Se compone de un subsidio ordinario que data desde los años noventa en que se inicia el programa de Becas al Desempeño Académico, pero se incrementa con base a la plantilla de personal académico de las Universidades Públicas Estatales (UPEA) que la SEP reconoce desde 2002 (Ruiz de la Torre y López, s/f).

Tiene la especificidad de estar orientado a los académicos cuya principal actividad es la docencia frente a grupo, que a su vez cumplan con los requisitos establecidos por cada reglamentación institucional. Los estímulos que se asignan en principio son al personal de carrera de tiempo completo con categoría de técnico y profesor de carrera asociados y titulares, pero se pueden incluir en el programa al personal de medio tiempo, tres cuartos de tiempo y de asignatura cuando las IES cuenten con recursos adicionales provenientes de diversas fuentes (aportaciones del gobierno estatal, ingresos propios, etc.). El incentivo se otorga por un año fiscal, el monto se ajusta a variaciones del salario mínimo vigente en el Distrito Federal y de acuerdo a diferentes niveles tabulares, según la puntuación alcanzada en los tres factores evaluados: a) calidad en el desempeño de la docencia, b) la dedicación a la docencia y, c) la permanencia en las actividades de docencia. Además, contempla mantener el apoyo para aquellos profesores que se encuentren en actividades de superación académica (SHCP, 2002).

Actualmente el denominado Programa de Carrera Docente en México comprende al conjunto de programas y acciones de fomento y estímulo que las IES (y otros niveles educativos) operan para sus académicos con diferentes denominaciones y normatividades según la institución de que se trate.

Durante los años noventa también se pusieron en marcha otros programas para mejorar la preparación del profesorado universitario al reconocerse en el marco del PME la necesidad de mejorar el nivel de habilitación de la planta académica y con ello mejorar su desempeño y elevar la calidad de la educación. Se crea así en 1994 el Programa Nacional 
de Superación Académica (SUPERA)², como una iniciativa conjunta de la ANUIES y la Subsecretaría de Educación Superior (SES). Constituyó el primer esfuerzo de alcance nacional orientado a impulsar la formación al nivel de posgrado de los profesores universitarios, pretendió fundamentalmente "incrementar de manera significativa en un horizonte de 6 años la proporción de académicos de carrera con especialidades, maestrías y doctorados" (Pallán, 1994, p. 2), con aportaciones del Gobierno Federal. Planteó la necesidad de aplicar criterios de equidad y un enfoque compensatorio en el que se apoyó preferentemente a las instituciones afiliadas a la ANUIES inicialmente, y fortalecer y consolidar programas de posgrado. Se mantuvo vigente hasta el año 2000 bajo la conducción de la ANUIES.

Durante su vigencia se formaron cerca de 2000 profesores en activo en las IES en instituciones nacionales y extranjeras (Comas-Rodríguez y Rivera, 2011). El programa "supuso el compromiso de las instituciones de no contratar personal sin estudios de posgrado, y apoyos para la apertura de plazas de tiempo completo" (Canales y Gilio, 2008, 22).

Simultáneamente a la aplicación del programa SUPERA surgen otras iniciativas institucionales de superación académica para ampliar su cobertura, la más importante a nivel nacional es el Programa de Mejoramiento del Profesorado (PROMEP), creado en 1996 en el marco del Programa de Desarrollo Educativo (PDE) 1995-2000 y como "respuesta a las recomendaciones que la Organización de la Naciones Unidas para la Educación, la Ciencia y la Cultura (UNESCO) realizó en 1966, en ellas se menciona que el país necesitaba crear un sistema de profesionalización docente con las capacidades de investigación-docencia típicas de la educación superior en el mundo" (SEP, 2015b, 15) que destaca el papel central que desempeñan los profesores para lograr que la educación superior en México alcance los niveles de calidad internacional.

Producto de la colaboración de la ANUIES, CONACYT, la Subsecretaría de Educación e Investigación Tecnológicas (SEIT) y la Subsecretaría de Educación Superior y Científica (SESIC), el PROMEP tiene como finalidad última “... sustentar la mejor formación de los estudiantes de educación superior, universitaria y tecnológica; para ello se plantea como principio la sólida formación académica del profesorado y su articulación en cuerpos académicos comprometidos con sus instituciones y articulados a los medios nacionales e internacionales de generación y aplicación del conocimiento. Al elevar la calidad del profesorado y mejorar su dedicación a las tareas académicas fundamentales, centradas en la figura del profesor de tiempo completo como docenteinvestigador, se reforzará la dinámica académica que constituye la columna vertebral de la educación superior" (PROMEP, 1996, p. 1).

A diferencia de las políticas de apoyo a la formación de los académicos como el SUPERA y las que otorgara el CONACYT desde los años 90 que amplía sus becas para posgrados nacionales e internacionales, el PROMEP no solo confiere becas para estudiar un posgrado, sino también recursos para la reincorporación del personal académico y para

\footnotetext{
${ }_{2}$ Su antecedente es el Programa Nacional de Formación de Profesores (1972-1982), financiado por el gobierno federal y operado por la ANUIES.

${ }^{3}$ Diario Oficial, Decimoprimera Sección, jueves 31 de diciembre, 2015b, p. 15.
} 
iniciar proyectos de investigación, además enfatiza que el académico que termine un posgrado sea contratado en una categoría superior, se considere asimismo el perfil PROMEP como prerrequisito en las nuevas contrataciones.

Este perfil PROMEP deseable se refiere al académico que además de poseer un grado académico mayor al del programa de estudios que imparte, en el caso de los profesores de tiempo completo, distribuya su tiempo de manera equilibrada en las funciones de docencia, investigación, tutorías y gestión académica. Además, deberá formar parte de un cuerpo académico consolidado que tenga estudios de posgrado, desarrollar sus funciones en condiciones de infraestructura adecuadas, y colaborar en redes internacionales académicas (PROMEP, 1996).

Con algunos ajustes, principalmente de tipo operativo, en 2014 el PROMEP cambió al Programa para el Desarrollo Profesional Docente (PROPED), actualmente vigente. El programa retoma los principios, criterios, apoyos y reconocimientos que se otorgaban a través del PROMEP, además de ampliarse a la educación básica y media superior. Sin embargo, aclara que para el caso de las universidades públicas estatales, de apoyo solidario y federales, el reconocimiento se otorgará a "Profesores/as de Tiempo Completo con perfil deseable, que se refiere al profesor/a universitario que posee un nivel de habilitación académica superior al nivel de los programas educativos que imparte, preferentemente cuenta con el doctorado y, además, realiza de forma equilibrada actividades de docencia, generación o aplicación innovadora de conocimientos, tutorías y gestión académica-vinculación” (SEP, 2015b, 15)

Si bien el PROMEP es el vértice de las políticas federales para la superación académica del profesorado universitario y evaluación de su desempeño, el Programa Integral de Fortalecimiento Institucional (PIFI), implementado por el Gobierno Federal a través de la SEP desde 2001, es también la política más importante orientada a la evaluación institucional de las universidades públicas mexicanas, y en este sentido acompaña al PROMEP en tanto que en sus propósitos explícitos se encuentran identificados los programas de compensación salarial. El programa incentiva procesos integrales de planeación participativa cuyo propósito es fomentar la mejora continua de la calidad de los programas y servicios educativos, a través de la asignación de fondos extraordinarios bajo concurso (SEP, 2011).

Las universidades se proponen contribuir al mejoramiento de la calidad con la formulación de proyectos institucionales (los PIFI), los cuales son evaluados en términos de la habilitación de los principales actores -profesores y estudiantes- y la evaluación y acreditación de programas (Buendía, 2013). Por lo que un aspecto central para la obtención de recursos financieros, tanto para infraestructura como para actividades académicas (cursos, profesores invitados, viajes, etc.) es la planeación minuciosa del desarrollo de los perfiles deseables y cuerpos académicos por Dependencia de Educación Superior (De Vries, González, León y Hernández, 2008).

${ }^{4}$ Sus reglas de operación para el año 2016 se emiten en el Diario Oficial, Decimoprimera Sección, jueves 31 de diciembre, 2015 b, p. 15 . 


\section{Programas de evaluación y estímulos a la productividad académica en la UNAM}

El caso de la Universidad Nacional Autónoma de México (UNAM) consituye un caso particular tanto por el tratamiento gubernamental hacia la institución, como por la amplia y conocida participación de sus académicos ante los programas de evaluación e incentivos al trabajo académico, que se evidencia en las acciones de negociación por la homologación salarial de estos programas, y la inclusión de las AAPAUNAM ${ }^{5}$ como interlocutor en el seguimiento de las mismas, al menos entre 1993 y 1996 (Canales, 2001).

En su caso, los programas de Becas al Desempeño Académico (para Asociados y Titulares) inscritos en la línea de Evaluación a la Productividad Individual, se originan en el mismo marco que los programas ya descritos, caracterizado por las exigencias para mejorar la preparación del profesorado universitario y por la situación prevaleciente en el conjunto de la educación superior mexicana. Estos programas tienen su concreción en el proyecto de "academización" de la UNAM, propuesto durante el rectorado del Dr. José Sarukhán (1989-1996) que respondía en parte al propósito de revertir la baja profesionalización de una parte importante del personal académico, lo cual se buscó mediante la habilitación de varios programas orientados a la superación académica, el fortalecimiento de condiciones propicias a la incorporación de nuevos académicos, el fomento de estímulos o becas basados en la evaluación del rendimiento individual de los investigadores y profesores (Arenas, 1998).

En este contexto, en 1990 se crea el Programa de Estímulos a la Productividad y al Rendimiento del Personal Académico de Carrera (PEPRAC), orientado a otorgar un estímulo académico a los profesores de carrera y como estrategia para incentivar la superación del personal académico, aumentar su participación en la formación de recursos humanos, estimular la vinculación docencia-investigación, premiar los altos niveles de productividad y calidad académica, y propiciar la permanencia en la Institución. El programa otorgó un estímulo mensual con vigencia de un año y opción a ser renovado, de acuerdo a la productividad académica del personal de carrera de tiempo completo. El sistema de evaluación consideró la formación y escolaridad, las aportaciones a las labores docentes y la productividad y calidad del desempeño, a los que se les otorgó pesos diferenciales.

En el periodo de 1990 a 1994 se introdujeron cambios importante al programa, como la precisión de las compensaciones que debían corresponder a profesores, investigadores y técnicos académicos, se integraron especificaciones de acuerdo a la naturaleza de los subsistemas de la UNAM (bachillerato, escuelas, facultades, centros), se aumentaron de 4 a 6 los niveles de asignación y sus montos con base en un número de salarios mínimos para cada uno, y se amplió de uno a dos años la permanencia del personal en el programa (Malo y Rojo, 1996).

5 Asociación Autónoma del Personal Académico de la Universidad Nacional Autónoma de México (AAPAUNAM) Sindicato u organismo representativo de los académicos universitarios. 
Después de revisar los puntos críticos del PEPRAC, y considerando las objeciones y percepciones de los académicos adscritos al programa, en 1993 el rector anuncia nuevas acciones para resarcir de manera gradual y mediante diversas acciones el deterioro salarial que los académicos soportaron durante la década de los ochenta, ${ }^{6}$ y en 1994 se estable un nuevo programa denominado Primas al Desempeño Académico del Personal de Tiempo Completo (PRIDE), que enfatiza "reconocer la labor de los académicos de tiempo completo que hayan realizado sus labores de manera sobresaliente; propiciar que éstas conjuguen la formación de recursos humanos, la docencia frente a grupo, la investigación y la extensión académica; así como fomentar la superación del personal académico y elevar el nivel de productividad y calidad de su desempeño" 7

A diferencia del PEPRAC, la prima de los académicos es equivalente a un porcentaje del salario tabular vigente del académico, que tiene repercusión en la prima de antigüedad docente, y puede ser otorgada en cuatro niveles, del A al D cuyos porcentajes correspondientes van del $45 \%$ al $105 \%$, y varían de acuerdo al tipo de dependencia de la UNAM (escuelas, facultades o multidisciplinarias). La evaluación es por pares académicos, integrados en comisiones evaluadoras y ha logrado la deshomologación de ingresos para el personal de tiempo completo.

El PRIDE ha tenido varios ajustes en los últimos años, como la duración del estímulo que se ha ampliado de 3 a 5 años para todos los niveles, así como la opción de solicitar por parte de los académicos de 60 a 69 años de edad, la exención de la evaluación en el programa y conservar su nivel de estímulo evaluado con un monto fijo y no como porcentaje del salario tabular, o bien, se ha establecido la obligatoriedad de esta condición para los académicos de 70 años y hasta su retiro. ${ }^{8}$

Como parte de la ampliación de la política institucional de estímulos al personal académico de la UNAM, en 1993 se anuncia la extensión del programa y se crea el Programa de Estímulos a la Productividad y al Rendimiento del Personal Académico de Asignatura (PEPASIG), que se dirige a personal académico de asignatura que no tienen nombramiento de profesor o investigador de carrera, y a técnicos académicos con actividad docente frente a grupo.

El programa estimula la labor de los profesores que cuenten con un año de antigüedad, que hayan realizado sus actividades académicas de manera sobresaliente, y hayan elevado su nivel de productividad y calidad de su desempeño. El estímulo consiste en el pago de una cantidad fija mensual por cada por cada tres horas semanales de clase frente a grupo, se otorga durante un año e individualmente, y se distinguen tres niveles de acuerdo al grado académico. La evaluación de los profesores de asignatura corresponde a los Consejos Técnicos de las entidades académicas. ${ }^{9}$

En la tabla 1 se presenta un resumen de los programas descritos.

\footnotetext{
6 “Anuncia el Rector nuevos estímulos para académicos”, Gaceta UNAM, 15 marzo de 1993, pp. 1-5.

7 Convocatoria. Programa de Primas al Desempeño del Personal Académico de Tiempo Completo (PRIDE), Gaceta UNAM, noviembre 2001, pp. 18-20.

${ }^{8}$ Convocatoria PRIDE 2017- DGAPA-UNAM.

${ }^{9}$ PEPASIG. DGAPA-UNAM.
} 
Tabla1. Programas de evaluación e incentivos al trabajo académico en las instituciones de educación superior públicas en México

\begin{tabular}{|c|c|c|c|c|c|}
\hline SIGLAS & PROGRAMA & $\begin{array}{c}\text { ORIGEN/ } \\
\text { INSTANCIA }\end{array}$ & VIGENCIA & PROPÓSITO & COBERTURA \\
\hline SNI & $\begin{array}{c}\text { Sistema } \\
\text { Nacional de } \\
\text { Investiga-dores }\end{array}$ & $\begin{array}{c}\text { CONACYT } \\
\text { Responder a la crisis } \\
\text { económica de 1982, y } \\
\text { fomentar el desarrollo } \\
\text { científico y } \\
\text { tecnológico } \\
\end{array}$ & $\begin{array}{l}\text { 1984/ } \\
\text { vigente }\end{array}$ & $\begin{array}{c}\text { Reconocer a los } \\
\text { investigadores en activo } \\
\text { mediante el otorgamiento de } \\
\text { recursos adicionales por } \\
\text { productividad en la } \\
\text { investigación }\end{array}$ & NACIONAL \\
\hline PBD & $\begin{array}{l}\text { Programa } \\
\text { Nacional de } \\
\text { Becas al } \\
\text { Desempeño } \\
\text { Académico (de } \\
\text { Carrera } \\
\text { docente) }\end{array}$ & $\begin{array}{c}\text { SEP } \\
\text { Responder a la } \\
\text { problemática salarial } \\
\text { del personal } \\
\text { académico de las IES, } \\
\text { y promover la } \\
\text { permanencia y } \\
\text { promoción del trabajo } \\
\text { académico en docencia } \\
\text { e investigación }\end{array}$ & $\begin{array}{l}1990-1992 \\
1992-1994\end{array}$ & $\begin{array}{l}\text { Revalorar el desempeño de } \\
\text { los docentes de tiempo } \\
\text { completo mediante } \\
\text { incentivos económicos, a fin } \\
\text { de incrementar la } \\
\text { permanencia, calidad y } \\
\text { desempeño integral de las } \\
\text { actividades académicas } \\
\text { (investigación, tutorías, } \\
\text { acciones de servicio y } \\
\text { docencia) }\end{array}$ & NACIONAL \\
\hline $\begin{array}{l}\text { ESDE- } \\
\text { PED }\end{array}$ & $\begin{array}{l}\text { Programa de } \\
\text { Estímulos al } \\
\text { Desempeño del } \\
\text { Personal SEP- } \\
\text { Docente }\end{array}$ & $\begin{array}{c}\text { SEP-SHCP } \\
\text { Resultado de la fusión } \\
\text { del } \\
\text { Programa de Becas al } \\
\text { Desempeño } \\
\text { Académico y el } \\
\text { Programa de Carrera } \\
\text { Docente }\end{array}$ & $\begin{array}{c}1994 / \\
\text { vigente }\end{array}$ & $\begin{array}{c}\text { Estimular el desarrollo del } \\
\text { personal académico dedicado } \\
\text { a la 196ocência, mediante } \\
\text { incentivos económicos, a fin } \\
\text { de elevar la calidad, } \\
\text { dedicación y docência, } \\
\text { investigación, tutorías y } \\
\text { participación en cuerpos } \\
\text { colegiados } \\
\end{array}$ & NACIONAL \\
\hline $\begin{array}{l}\text { SUPE- } \\
\text { RA }\end{array}$ & $\begin{array}{c}\text { Programa de } \\
\text { Superación del } \\
\text { Personal } \\
\text { Académico }\end{array}$ & $\begin{array}{c}\text { ANUIES y SES } \\
\text { Responder a la } \\
\text { necesidad de ampliar y } \\
\text { mejorar la formación } \\
\text { del personal } \\
\text { académico de las IES y } \\
\text { mejorar los programas } \\
\text { de posgrado }\end{array}$ & $1994-2000$ & $\begin{array}{l}\text { Incrementar de manera } \\
\text { significativa en un periodo de } \\
6 \text { años la proporción de } \\
\text { académicos de carrera de las } \\
\text { IES con especialidades, } \\
\text { maestrías y doctorados, } \\
\text { mediante la aportación de } \\
\text { recursos económicos a las } \\
\text { IES }\end{array}$ & NACIONAL \\
\hline $\begin{array}{l}\text { PRO- } \\
\text { MEP }\end{array}$ & $\begin{array}{l}\text { Programa de } \\
\text { Mejoramiento } \\
\text { del Profesorado }\end{array}$ & $\begin{array}{c}\text { ANUIES, } \\
\text { CONACYT, SEIT Y } \\
\text { SESIC } \\
\text { Responder a las } \\
\text { recomendaciones de la } \\
\text { UNESCO (1966) ya la } \\
\text { necesidad de elevar y } \\
\text { ampliar la cobertura } \\
\text { en la formación del } \\
\text { personal académico de } \\
\text { las IES y alcanzar los } \\
\text { niveles de calidad } \\
\text { internacional en la } \\
\text { educación superior }\end{array}$ & $1996-2014$ & $\begin{array}{l}\text { Habilitar sólidamente a los } \\
\text { profesores de las IES para su } \\
\text { incorporación a cuerpos } \\
\text { colegiados y su articulación a } \\
\text { medios nacionales e } \\
\text { internacionales académicos y } \\
\text { científicos, mediante } \\
\text { otorgamiento de becas para } \\
\text { estudios de posgrado y } \\
\text { recursos económicos para su } \\
\text { reincorporación, promoción } \\
\text { e iniciación de proyectos de } \\
\text { investigación }\end{array}$ & NACIONAL \\
\hline $\begin{array}{l}\text { PRO- } \\
\text { PED }\end{array}$ & $\begin{array}{l}\text { Programa para } \\
\text { el Desarrollo } \\
\text { Profesional } \\
\text { Docente }\end{array}$ & $\begin{array}{c}\text { SEP } \\
\text { Sustituir al PROMEP } \\
\text { bajo los mismos } \\
\text { lineamientos, y amplía } \\
\text { su cobertura a la } \\
\text { educación básica y } \\
\text { media superior }\end{array}$ & $\begin{array}{c}\text { 2014/vigen } \\
\text { te }\end{array}$ & $\begin{array}{c}\text { Habilitar y reconocer a los } \\
\text { profesores de las IES que } \\
\text { cumplan un "perfil académico } \\
\text { deseable” que realiza } \\
\text { actividades de docencia, } \\
\text { generación o aplicación } \\
\text { innovadora de } \\
\text { conocimientos, tutorías y } \\
\text { gestión académica- } \\
\text { vinculación, de forma } \\
\text { equilibrada, mediante el } \\
\text { otorgamiento de becas e } \\
\text { incentivos económicos }\end{array}$ & NACIONAL \\
\hline
\end{tabular}


Tabla1. Programas de evaluación e incentivos al trabajo académico en las instituciones de educación superior públicas en México (continuación)

\begin{tabular}{|c|c|c|c|c|c|}
\hline SIGLAS & PROGRAMA & $\begin{array}{c}\text { ORIGEN/ } \\
\text { INSTANCIA }\end{array}$ & VIGENCIA & PROPÓSITO & COBERTURA \\
\hline $\begin{array}{c}\text { PE- } \\
\text { PRAC }\end{array}$ & $\begin{array}{c}\text { Programa de } \\
\text { Estímulos a la } \\
\text { Productividad y } \\
\text { al Rendimiento } \\
\text { del Personal } \\
\text { Académico de } \\
\text { Carrera }\end{array}$ & $\begin{array}{c}\text { UNAM } \\
\text { Revertir la baja } \\
\text { profesionalización del } \\
\text { personal académico, } \\
\text { propiciar su } \\
\text { permanencia en la } \\
\text { Institución y elevar los } \\
\text { niveles de } \\
\text { productividad, } \\
\text { superación académica y } \\
\text { calidad en su } \\
\text { desempeño, en } \\
\text { profesores de carrera de } \\
\text { tiempo completo }\end{array}$ & 1990-1994 & $\begin{array}{l}\text { Incentivar la superación } \\
\text { académica del personal } \\
\text { académico, aumentar su } \\
\text { participación en la } \\
\text { formación de recursos } \\
\text { humanos, estimular la } \\
\text { vinculación docencia- } \\
\text { investigación, y premiar los } \\
\text { altos niveles de } \\
\text { productividad y calidad } \\
\text { académica, mediante el } \\
\text { otorgamiento de estímulos } \\
\text { económicos o becas basados } \\
\text { en la evaluación del } \\
\text { rendimiento individual }\end{array}$ & UNAM \\
\hline PRIDE & $\begin{array}{l}\text { Primas al } \\
\text { Desempeño } \\
\text { Académico del } \\
\text { Personal de } \\
\text { Tiempo } \\
\text { Completo } \\
\text { (PRIDE) }\end{array}$ & $\begin{array}{c}\text { UNAM } \\
\text { Sustituir al PEPRAC } \\
\text { bajo los mismos } \\
\text { lineamientos y } \\
\text { compensar de manera } \\
\text { gradual el deterioro } \\
\text { salarial del personal } \\
\text { académico de carrera de } \\
\text { tiempo completo }\end{array}$ & $1994 /$ vigente & $\begin{array}{l}\text { Reconocer la labor de los } \\
\text { académicos de tiempo } \\
\text { completo que hayan } \\
\text { realizado sus labores de } \\
\text { manera sobresaliente; } \\
\text { propiciar que éstas } \\
\text { conjuguen la formación de } \\
\text { recursos humanos, la } \\
\text { docencia frente a grupo, la } \\
\text { investigación y la extensión } \\
\text { académica, mediante la } \\
\text { asignaciones porcentuales } \\
\text { sobre el salario base y la } \\
\text { antigüedad del personal } \\
\text { académico } \\
\end{array}$ & UNAM \\
\hline $\begin{array}{l}\text { PEPA- } \\
\text { SIG }\end{array}$ & $\begin{array}{l}\text { Programa de } \\
\text { Estímulos a la } \\
\text { Productividad y } \\
\text { al Rendimiento } \\
\text { del Personal } \\
\text { Académico de } \\
\text { Asignatura }\end{array}$ & $\begin{array}{c}\text { UNAM } \\
\text { Ampliar la política } \\
\text { institucional de } \\
\text { estímulos al personal } \\
\text { académico de } \\
\text { asignatura con } \\
\text { actividad docente frente } \\
\text { a grupo }\end{array}$ & $1995 /$ vigente & $\begin{array}{l}\text { Estimular la labor de los } \\
\text { profesores que hayan } \\
\text { realizado sus actividades } \\
\text { académicas de manera } \\
\text { sobresaliente, y hayan } \\
\text { elevado su nivel de } \\
\text { productividad y calidad de } \\
\text { su desempeño, mediante el } \\
\text { otorgamiento de un } \\
\text { incentivo fijo mensual de } \\
\text { acuerdo a las horas semanas } \\
\text { frente a grupo y grado } \\
\text { académico }\end{array}$ & UNAM \\
\hline
\end{tabular}

Fuente: Elaboración propia

Estos programas considerados como "pagos por méritos" para las instituciones de educación superior se llegan a combinar con el Sistema Nacional de Investigadores (SNI), que como se comentó anteriormente, es un programa que opera a nivel nacional, y juntos pueden llegar a representar proporciones muy significativas, superiores al 60 por ciento del ingreso respecto al salario base de los académicos, si en el mejor de los casos se pertenece a las categorías y niveles más altos de estos programas. Además, hay que considerar la diferenciación que se produce por la antigüedad y el tipo de nombramiento, por ejemplo, el nombramiento de Titular C en el caso de la UNAM tiene el salario base más alto, por lo que estos programas han producido una amplia estratificación del personal académico al considerar sus ingresos (Ordorika y Navarro, 2006).

Es así que a partir de estos programas se ha generado un proceso de profesionalización de la carrera académica que ha venido a repercutir no sólo en la conducción y 
funcionamiento de cada institución, sino también en la reorientación de las actividades y tareas de los académicos al tener un impacto importante en su recuperación salarial.

La investigación que se ha derivado en este aspecto, en general indica que pocos académicos quedan indiferentes a los programas de estímulos e incentivos pues se juega en ello no sólo el incremento económico vinculado a mejores condiciones de vida, sino también simbolizaciones que tienen que ver con sus trayectorias sociales y académicas, su identidad profesional e institucional, su estatus y prestigio académico (Díaz Barriga, 1997; Furlán, 1998; García, 1998; Rueda, 1998).

Sin embargo, uno de los cuestionamientos cardinales que hacen varios estudiosos sobre el tema es si los programas de pagos por méritos o "bonos por evaluación" han contribuido a mejorar la calidad de la docencia, además de las actividades de investigación y extensión de la cultura de la educación superior, que conjuntamente con el mejoramiento de los salarios del personal académico, fueron las metas principales de estos programas (Acosta, 2004; Canales, 2001, 2008; Díaz Barriga y Díaz Barriga, 2008; Ordorika y Navarro, 2006; Rueda, 2011; Rueda y Torquemada, 2004).

\section{Aciertos, desaciertos y paradojas de los programas de evaluación académica desde la perspectiva de sus actores}

A continuación, se hace una síntesis de los hallazgos encontrados por varios investigadores, que en contextos particulares han tenido como interlocutores a los propios actores de la academia -profesores, funcionarios, administradores y estudiantes-, y han obtenido información relevante sobre los beneficios y problemas por demás complejos y polémicos a los que han dado lugar los programas de estímulos a la productividad académica.

\subsection{Desarrollo profesional y prestigio diferencial de los académicos}

De los aciertos más señalados, se argumenta que el incremento salarial asociado a la evaluación académica en las instituciones de educación superior públicas ha jugado un papel fundamental en el desarrollo e institucionalización de la vida académica, en tanto se ha logrado la elevación de los indicadores a las que están sometidas. Entre los cambios más significativos está el desarrollo profesional de los académicos, como es el incremento en la obtención de posgrados y de sus actividades y tareas, principalmente las que se priorizan en la evaluación de los programas como son: la impartición de conferencias, asistencia a congresos y presentación de trabajos, titulación de estudiantes, asesorías y tutorías, publicación de artículos, participación en programas institucionales y pertenencia a grupos académicos colegiados consolidados (Acosta, 2004 ${ }^{10}$; Díaz Barriga y Díaz Barriga, 2008; ${ }^{11}$ Patrón y Cisneros-Cohernour, 2014 ${ }^{12 ;}$ Silva, 2007 ${ }^{13}$ ).

\footnotetext{
10 Toma como punto de referencia el caso de la Universidad de Guadalajara (UDG).

${ }_{11}$ Aplicaron 97 entrevistas a diferentes actores de 17 instituciones de educación superior: principalmente a responsables institucionales (rectores, secretarios generales, directores de planeación, de facultades, escuelas, departamentos, centros o institutos), sujetos de la evaluación (académicos de tiempo completo: investigadores y profesores), y evaluadores (especialistas teóricos y expertos que realizan la evaluación)
} 
No obstante, las apreciaciones entre los actores llegan a ser contrastantes pues éstas dependen de la posición que ocupen y de las responsabilidades en las que participan para la operación de dichos programas. Para algunos funcionarios y directores (responsables de la organización y operación de los programas) el efecto principal de los programas de incentivos a la productividad académica ha sido un incremento sustancial de profesores con posgrado, así como la obtención de estatus y reconocimiento. Pero al mismo tiempo, otros apuntan que tales beneficios se dan a título personal, en función de los méritos personales, no en la institución en su conjunto (Díaz Barriga y Díaz Barriga, 2008), otros piensan que los programas de estímulos conllevan "perdedores" y "ganadores", por lo que también hay adhesiones y simpatías entre algunos académicos (Acosta, 2004); para otros más, se presentan inequidades al existir diferentes tipos de contrataciones en tanto los profesores de carrera tienen mayor carga docente que los investigadores (Patrón y Cisneros-Cohernour, 2014).

La percepción de inequidad entre los profesores de carrera es reportada de manera consistente en las investigaciones sobre el tema, en tanto se perciben en constante desventaja en relación con los investigadores para competir por un estímulo adicional al salario (Cordero, Galaz, Sevilla, Nishikawa y Gutiérrez, 2003; ${ }^{14}$ Estrada y Guillermo, 2005 ${ }^{15}$; Silva, 2007). El problema estriba en el grado de involucramiento del académico en las actividades de docencia e investigación y la importancia que se le otorga al desempeño de cada función, según el programa de estímulos que opere y la forma en que cada institución lo adopte.

El reconocimiento diferencial entre docencia e investigación ha sido tema de debate que va más allá del ámbito de los programas de estímulos a la productividad académica, sin embargo, en éstos se evidencia su complejidad y paradojas, pues aún y cuando algunos administradores o funcionarios reconocen la heterogeneidad del trabajo académico e intentan adecuar los programas de estímulos a las diversas realidades institucionales, disciplinarias o como respuesta a las inconformidades de los académicos, no obstante, su vínculo como condicionamiento salarial continúa generando percepciones de inequidades o injusticias entre los académicos porque en ello se juega no solo el porcentaje de un ingreso adicional sino también su estatus o prestigio profesional. Se ha encontrado, por ejemplo, que las propuestas de evaluar por separado a docentes e investigadores son bien recibidas por los docentes, mientras que, para los investigadores, obtener la misma beca que un académico dedicado fundamentalmente a la docencia, es minar el reconocimiento

\footnotetext{
12 Entrevistaron a 35 profesores de una universidad pública de Campeche y a un funcionario de la comisión a cargo de la evaluación de una universidad pública de Yucatán, quién a su vez analizó los resultados de una investigación sobre las percepciones de 137 docentes acerca del sistema de evaluación aplicada en 2005.

${ }^{13}$ Aplicó 27 entrevistas no-estructuradas a docentes de tiempo completo (que participan en el programa de estímulos de los cuatro institutos de la Universidad Autónoma de Ciudad Juárez (UACJ). También recolectó inconformidades y sugerencias del foro de estímulos de 2003 de la página electrónica de la UACJ.

14 Entrevistaron a 41 académicos de diversas áreas de conocimiento de tres unidades regionales de la Universidad Autónoma de Baja California (UABC) sobre el Programa de Estímulo al Personal Académico de la institución. Evalúan el cambio del modelo inicial de evaluación en el que se definía al académico ideal como un sujeto integral que desarrollaba por igual todas las funciones de la vida universitaria, por un modelo o sistema diferenciado para evaluar por separado a docentes e investigadores y comparar el desempeño de cada uno contra su propio grupo.

15 Aplicaron una encuesta a una muestra estratificada de 137 académicos de la Universidad Autónoma de Yucatán para conocer su opinión sobre la implementación del programa de estímulos.
} 
institucional a la investigación (Cordero, Galaz, Sevilla, Nishikawa y Gutiérrez, 2003). Asimismo, los profesores con un perfil de investigadores muestran inconformidad con las actuales cargas académicas al ponderarse la docencia frente a grupo en detrimento de sus actividades de investigación para poder acceder a los programas (Camarillo y Rincones, 2012) ${ }^{16}$.

Si bien se puede observar que a casi tres décadas de su operación, los programas de estímulos a la productividad académica han producido cambios significativos en el desarrollo profesional de los académicos, los programas a la productividad académica son percibidos tanto positivamente (vinculan la docencia e investigación, incentivan la promoción académica, buscan la excelencia a partir de posgrados) como por su carácter instrumental, en tanto el cuestionamiento que gira a nivel institucional es solamente en torno al mejor procedimiento o instrumento que permita evaluar la productividad y asociarla a un determinado monto económico, mientras que la percepción generalizada entre los académicos es que la instauración de los programas de estímulos ha creado una serie de conflictos y tensiones en las comunidades académicas.

\subsection{Instauración de una cultura "meritocrática", burocrática e individualista en los centros universitarios}

En una encuesta levantada en 2007 a nivel nacional por Galaz, Gil, Padilla, Sevilla y Martínez (2012), con la colaboración de diferentes universidades públicas y privadas, y de la Red de Investigadores sobre Académicos (RDISA), para evaluar las transformaciones que ha venido experimentado la profesión académica en varias dimensiones, analizan las tensiones y transformaciones que enfrentan los académicos en torno a su rol fundamental de docencia, investigación, gestión, vinculación y extensión, relacionadas a recompensas económicas que están diferenciando de manera negativa sus funciones.

La lógica de un sistema por puntajes o caracterizado principalmente por la cuantificación de los productos obtenidos para la evaluación de los académicos, y el hecho de asociar a la evaluación con la remuneración afecta la naturaleza del trabajo académico, ya que propicia que los docentes e investigadores respondan principalmente a las actividades que se traducen en los mejores ingresos por sobre otros criterios, como pudieran ser los derivados del proyecto educativo institucional, con lo que se corre el riesgo del quebrantamiento de la identidad institucional y académica entre sus profesores (Covarrubias, 2003). ${ }^{17}$

El tipo de relaciones que se han generado al interior de los claustros universitarios y la rivalidad que parece existir entre áreas de conocimiento (científicos de disciplinas duras vs disciplinas blandas), niveles y divisiones académicas (académicos de licenciatura vs de posgrado), incluso entre figuras académicas (profesores, investigadores, técnicos

\footnotetext{
${ }^{16}$ Entrevistaron a 11 académicos y tres funcionarios universitarios de la Universidad Autónoma de Ciudad Juárez (UACJ), para ver cómo influyen el SNI, el PROMEP y el ESDEPED en la vida académica de los profesores universitarios

17 Aplicó entrevistas a profundidad a 15 académicos de la carrera de psicología de la FES Iztacala-UNAM, con la intención de indagar los factores que impedían llegar a consensos entre la comunidad académica que permitieran un cambio o actualización del plan de estudios.
} 
académicos), han generado malestar entre los académicos, pero también la competencia desleal y la falta de solidaridad y cooperación (Díaz Barriga y Díaz Barriga, 2008).

La solidaridad y cooperación se soslayan cuando se percibe que otros espacios y niveles académicos pueden redituar en beneficio propio. Así el ámbito de la licenciatura, por ejemplo, deja de tener significado en la búsqueda de mayor reconocimiento y prestigio académico, se trata más bien de armar liderazgos académicos entre posgrado e investigación, y la atención a los alumnos deja de ser una prioridad para los académicos. Se desestima así uno de los principales propósitos de la docencia en la licenciatura que es la formación de generaciones con conocimientos, habilidades y actitudes éticas que les permitan insertarse en un mundo de trabajo por demás competido; en tanto el prestigio académico se supedita a la obtención de posgrados, ocupación de mejores plazas y al desarrollo de la investigación: el estatus académico se alcanza en el posgrado y la investigación (Covarrubias, 2003; Díaz Barriga y Díaz Barriga, 2008).

Lo paradójico aquí es que aún y cuando las políticas nacionales han centrado su atención e inversión financiera en la promoción de la actividad de investigación y en el incremento en los niveles formativos en la planta académica de las universidades públicas de los estados, la docencia sigue siendo la actividad principal en tiempo de dedicación y preferencia para la mayor parte de los académicos de tiempo completo de la educación terciara mexicana, pues en promedio dedican 22 horas semanales a las actividades relacionadas con la docencia, según los datos obtenidos por la encuesta nacional de académicos de tiempo completo, realizada por la Red de Investigadores sobre Académicos (RDISA) (Estévez y Martínez, 2011; Galaz, Gil, Padilla, Sevilla, Arcos y Martínez, 2012).

Lo que se alcanza a interpretar con estos datos es que aunque las políticas de formación y evaluación de los académicos han promovido el aumento de índices de profesores con un nivel formativo más alto (maestría y doctorado) y un mayor porcentaje de profesores de tiempo completo en el posgrado, cumpliéndose así algunas de las metas de las políticas públicas de la educación superior, no obstante, los profesores parecen estar más preocupados o motivados por las ventajas económicas que por mejorar sus prácticas de docencia e investigación. Sin embargo, "todavía se desconoce hasta dónde las universidades que ahora tienen altos índices de profesores con título de posgrado han mejorado el desempeño de sus funciones substantivas" (Estévez, 2009, p. 237).

Otro aspecto que resulta paradójico o incongruente para programas que impulsan la productividad, es la exigencia de constancias para probar las tareas realizadas que para los académicos es parte de un sistema fundado en la desconfianza y en la rutina administrativa, lo consideran un instrumento burocrático que induce a la simulación, en tanto desde la administración -consciente o inconscientemente-, se profundiza en la inequidad entre el profesorado por medio de los ingresos (Silva, 2007).

$\mathrm{Al}$ respecto, Ibarra y Porter (2007) anotan que el conjunto de prácticas de evaluación desde las que se controla a las universidades y a los académicos han desarticulado su tejido social en tanto que “... los modos de regulación académica basados en el control y la recompensa o el castigo, dificultan crecientemente la consolidación de una comunidad académica que fundamente su convivencia a partir del cultivo de la inteligencia, la reflexión y la ética” (p. 70). 
Lo cierto es que los procesos de diferenciación, que es una característica sustantiva del sistema de merit pay, al importarse de un enfoque empresarial ha irrumpido en los últimos años en las universidades de América Latina y de México en particular, y ha impactado severamente su quehacer (Ordorika, 2004), que al parecer “... enfrenta a los académicos entre sí y erosiona el sentimiento de pertenencia a un proyecto colectivo (Ordorika y Navarro, 2006, p. 62).

\subsection{La identidad docente en riesgo}

Los académicos, tanto en su función docente como la de investigadores, se sitúan en el centro mismo de los quehaceres que tiene socialmente asignados la universidad. Son los académicos, a través de las instituciones de educación superior, los que se encargan directamente del proceso de enseñanza y evaluación del aprendizaje, es decir, están legitimados social e institucionalmente como los formadores de las nuevas generaciones y se espera lo hagan con integridad y ética. Sin embargo, las presiones que ocasionan los procesos de evaluación académica han repercutido en la vida académica de las universidades y en el tipo de interacciones que percibe el académico en un recinto universitario, que contrasta con la imagen que de la cultura de la universidad se tiene y conlleva a una confrontación o desvalorización de la identidad de los docentes.

Es así que mientras expertos y directivos llegan a pensar que la principal motivación de los académicos en los programas de estímulos es la retribución económica sin que medie una vinculación directa con su desarrollo e identidad profesional, en los académicos prevalece la sensación de estar constantemente subvalorados en un sistema del que se desconfía de ellos, que deriva comúnmente en frustración, sentimiento de inequidad o injusticia, o el sentirse vulnerados o amenazados (Díaz Barriga y Díaz Barriga, 2008). Al respecto, Becher (2001) considera que más que las compensaciones económicas o de otro tipo, la búsqueda de prestigio es la que mueve a los académicos “... lo más importante que busca el académico, no es el poder, tras el cual va el político, ni la riqueza, tras la cual va el hombre de negocios, sino la buena reputación" (p. 78).

Del mismo modo existe la idea entre las autoridades académicas que hay una brecha generacional entre los académicos en relación a su aceptación para la participación en los programas de estímulos; al parecer los académicos más jóvenes aceptan con mayor naturalidad la nueva cultura de la evaluación, mientras que los mayores de cuarenta años son más resistentes ya que ésta no es acorde a su visión y prácticas de antaño (Díaz Barriga y Díaz Barriga, 2008). Sin embargo, otros encuentran que desde el punto de vista de los profesores de nuevo ingreso, las políticas de evaluación representan menos exigencias para los profesores con mayor antigüedad en la institución, lo que para ellos significa cumplir con parámetros que antes no eran exigidos a los profesores para ingresar a las instituciones de educación superior (IES), incluso los profesores de mayor antigüedad emprenden consistentes estrategias para permanecer y escalar niveles dentro del SNI, en tanto a los de nuevo ingreso se les dificulta más tener acceso a ellas (Camarillo y Rincones, 2012).

Lo cierto es que los diversos puntos de vista que se tienen de los programas de evaluación académica están relacionados, además de la posición que se ocupe en las instituciones, con factores que van desde la trayectoria y etapa en la que se encuentra el académico en su carrera profesional, según la institución de adscripción laboral, la afiliación a una disciplina de conocimiento, las oportunidades que se ofrecen 
institucionalmente para acceder a plazas y ascender en nombramientos, hasta la edad y el tiempo que se lleva en la institución.

También los efectos estresantes que las exigencias del contexto universitario representan, asociado a las cargas de trabajo y las recompensas para los académicos, han sido documentados al describir el síndrome del desgaste profesional en académicos, que influye de manera diferencial según el contrato laboral, antigüedad, disciplina y género (Ramírez y Padilla, 2008). Se subraya asimismo que la racionalidad impuesta por las instituciones, asociada al individualismo y competitividad de excelencia, responde a un imaginario de trascendencia, "los académicos que producen" y "los académicos que no producen", pero que aunque conlleva el agotamiento físico y mental como una nueva forma de distinción y carrera académica dentro de las instituciones universitarias, conduce a los académicos a perseguir una imagen de sí mismos de conformidad a los estándares de excelencia y de triunfo en detrimento de su identidad constitutiva (Remedi, 2006). Se llegan incluso a producir efectos psicológicos, económicos y familiares cuando los académicos no logran ingresar o mantenerse en los programas de estímulos a la productividad, como sería el ingreso y/o permanencia en el SNI (Camarillo y Rincones, 2012).

Los escenarios delineados por las investigaciones citadas no parecen halagüeñas, en tanto son los académicos, tanto en su función docente como la de investigadores, los que se encargan directamente del proceso de enseñanza y evaluación del aprendizaje que lleva a la certificación de los aprendizajes obtenidos, por ello y como se advierte desde el Programa Nacional de Educación 2001-2006, falta documentar como prioridad en la agenda de la investigación educativa en este campo, lo que han representado los programas de estímulos a la producción académica en el mejoramiento de la calidad educativa.

Desde la perspectiva de los estudiantes existen algunos datos al respecto, en una investigación de De Vries, González, León y Hernández (2008) en la que indagaron las opiniones de los estudiantes sobre el desempeño de sus profesores, al considerar que son los principales evaluadores para juzgar éste, encontraron que contrario a lo que se esperaría a partir de lo postulado por las políticas de estímulos, el hecho de ser profesor de tiempo completo, con perfil PROMEP, con titularidad, ser miembro del SNI, y tener un alto nivel en el ESDEPED, no se traduce en una mejor calificación de docencia. Las pequeñas diferencias reconocidas entre distintos tipos de profesores se deben a otros factores, como el tamaño del grupo o el área de conocimiento.

\section{Consideraciones finales}

Mucho de lo que se ha escrito sobre los efectos de las políticas de evaluación ligadas primordialmente a la remuneración y sus efectos no deseados, se expresan de manera clara en las percepciones de los interlocutores de los investigadores que en este trabajo se recuperan, si bien la exploración realizada no ha sido exhaustiva, se observa consistencia, aún y las diferencias a favor o en contra, en las formas en que los académicos, autoridades y funcionarios piensan y viven los programas de evaluación académica. 
Los discursos de los actores de la evaluación académica, desafortunadamente confirman los argumentos de Ibarra (2007) en el sentido de que nos hemos transformado de "homo academicus" en "homo economicus", en tanto las funciones sustantivas de los académicos parecen estar más presididas por los cálculos en términos de qué tanto reditúa más la inversión del tiempo dedicado en la elaboración de artículos o desarrollo de proyectos de investigación, en comparación a la producción y generación de conocimiento, o en lo relativo a la formación y la docencia relacionadas a una educación de calidad. Como apuntan Ordorika y Navarro (2006):

... la división del trabajo académico ha dejado de estar sujeta al principio de la proliferación para ser regulada por el cumplimiento de múltiples funciones que distraen a los académicos y a sus instituciones de las tareas sustantivas. La evaluación, si bien abre posibilidades de acción, lo son dentro de márgenes prefijados en los programas de evaluación, de los cuales no es posible salir a riesgo de perder el juego. (p. 57)

Lo que es un hecho es que estos programas parecen haber llegado para quedarse, en tanto sus efectos más positivos se manifiestan en la mejora de las condiciones salariales de los académicos de tiempo completo de las IES nacionales, por lo que la evaluación del trabajo académico no parece rechazarse como tal, más bien, se refuta por la lógica irracional y la cuantificación con la que se realiza. Como argumenta Acosta (2004), el malestar que develan los académicos parece más deberse a:

la tensión que existe entre la acumulación de capital académico producto directo o indirecto de los estímulos, y el establecimiento o inexistencia de 'capital social universitario, es decir, de confianza en el sentido de las reglas que cauterizan la distribución de los incentivos. (p. 77)

Se trata más bien entonces de la necesidad de buscar alternativas que no trastoquen las funciones sustantivas de las IES, y de recuperar el trabajo colegiado en pro de una educación y producción académica de calidad. Se requieren políticas más certeras que reconozcan la productividad de la enseñanza y de la investigación de modo diferente en los distintos momentos de la carrera del académico, y contemplen la necesidad de que sus efectos reditúen prioritariamente en la elevación de la calidad educativa.

El escenario actual de los académicos mexicanos es complejo y plantea retos profundos por enfrentar. El reconocimiento de los problemas expuestos ha sido explícito por parte de las autoridades oficiales a partir del diagnóstico realizado en el 2001 en el que expresaron su preocupación por los efectos encontrados, externando la necesidad de atenderlos (SEP, 2001), no obstante, poco se han atendido los problemas que traen consigo basar la evaluación del desempeño de los académicos en la cuantificación de actividades y de productos, por el contrario, ésta se ha incrementado, un ejemplo es el uso de métricas de impacto en las publicaciones para evaluar su importancia, como si con ello se resolvieran los dilemas de objetividad en los juicios de calidad. Siguen pendientes la propuestas que permitan evaluar la calidad de la enseñanza, la calidad de la práctica docente en el aula, el impacto de las asesorías o tutorías a los estudiantes, entre otros.

Se requiere continuar con estudios sobre los procesos de profesionalización poniendo en claro las condiciones contextuales de los académicos, sus roles, compromisos y tareas particulares que tienen que realizar dentro de las universidades. Debe por lo tanto contemplarse que participen los actores de la evaluación, particularmente los académicos que han quedado excluidos de las decisiones en cuanto a procesos, requisitos, criterios, 
normatividad y reglamentos; en otras palabras, se necesita establecer un diálogo entre la academia y la administración.

Lo que preocupa es que la atención a las necesidades aún no resueltas con respecto a la evaluación de los académicos, se está quedando rezagada en tanto se presentan otros temas emergentes con respecto a la carrera académica que hoy son apremiantes, como son el envejecimiento docente, la jubilación de los académicos y las formas de adaptarse a los programas de estímulo. Grediaga y López (2011), advierten que en el transcurso de los últimos diez años existen cambios en la organización del trabajo y el uso del tiempo por los académicos ya que éstos han cambiado sus prácticas, por lo que la agenda de investigación sobre la carrera académica se centra hoy en varios aspectos relacionados con el empleo, la diferencia en las trayectorias académicas, la socialización de las nuevas generaciones, la jubilación, el retiro y la renovación de la planta. Por ahora estos temas se están trabajando principalmente desde los enfoques de la sociología de las profesiones, y los problemas de la evaluación de los académicos parecen haberse quedado en el tintero.

\section{Referencias}

Aboites, H. (1999). La cultura de la evaluación en México y la evaluación de los académicos. En M. Rueda y M. Landesmann (Coords.), ¿ Hacia una nueva cultura de la evaluación de los académicos? (pp. 34-55). Ciudad de México: CESU/UNAM.

Acosta, A. (2004). El soborno de los incentivos. En I. Ordorika (Coord.), La academia en jaque. Perspectivas políticas sobre la evaluación de la educación superior en México (pp. 75-89). Ciudad de México: CRIM-UNAM- Miguel Ángel Porrúa.

Arenas, J. (1998). Políticas de evaluación en la comunidad académica de México (1984-1997): El SNI y el Programa del PRIDE en la UNAM. (Tesis inédita de Maestría). Instituto de Investigaciones Dr. José María Luis Mora, México.

Becher, T. (2001). Tibus y territorios académicos. La indagación intelectual y la cultura de las disciplinas. Barcelona: Gedisa.

Buendía, A. (2013). Genealogía de la evaluación y acreditación de instituciones en México. Perfiles Educativos, 35(spe), 17-32.

Camarillo, H. M. y Rincones, R. (2012, agosto). La influencia actual de tres políticas públicas de evaluación académica en profesores de una universidad de la Frontera del Norte de México: el caso de la Universidad Autónoma de Ciudad Juárez. Ponencia presentada en el Segundo Congreso Interdisciplinario de Investigación en Educación. Universidad de Chile, Chile.

Canales, A. (2001). La experiencia institucional con los programas de estímulo: La UNAM en el periodo 1990-1996. Ciudad de México: DIE-CINVESTAV-IPN.

Canales, A. (2008). La evaluación de la actividad docente: a la espera de iniciativas. Revista Electrónica de Investigación Educativa, 10 (spe), 1-20.

Canales, A. y Gilio, M. C. (2008). La actividad docente en el nivel superior: ¿Diferir el desafío? En M. Rueda (Coord.), La evaluación de los profesores como recurso para mejorar su práctica (pp. 17-38). Ciudad de México: UNAM-Plaza y Valdés.

Cetina, E. (2004). Estímulos al desempeño del personal docente en las universidades públicas estatales. En M. Rueda (Coord.), ¿Es posible evaluar la docencia en la Universidad? 
Experiencias en México, Canadá, Francia, España y Brasil (pp. 77-82). Ciudad de México: ANUIES-Universidad Autónoma Benito Juárez de Oaxaca.

Comas-Rodríguez, O. y Rivera, A. (2011). La docencia universitaria frente a los estímulos económicos. Educación, Sociedad y Cultura, 32, 41-54.

CONACYT. (2017). Sistema Nacional de Invesgigadores. Recuperado de http://www.conacyt.gob.mx/index.php/el-conacyt/sistema-nacional-de-investigadores (3 mayo 2017)

Cordero, G., Galaz, J., Sevilla, J. J., Nishikawa, K. y Gutiérrez, E. (2003). La Evaluación de la heterogeneidad de los perfiles académicos por medio de un programa de estímulos al personal académico. Experiencia de una universidad pública estatal. Revista Mexicana de Investigación Educativa, 8(19), 759-783.

Covarrubias, P. (2003). Currículum, disciplina y profesión desde la perspectiva de los académicos de Psicología Iztacala. Tesis doctoral no publicada. UNAM, México.

De Vries, W. (1998). El exorcismo de diablos y ángeles. Los efectos de las políticas públicas sobre el trabajo académico. Tesis doctoral no publicada. Universidad Autónoma de Aguascalientes, México.

De Vries, W., González, G. León, P. y Hernández, I. (2008). Políticas públicas y desempeño académico, o cómo el tamaño sí importa. CPUe Revista de Investigación Educativa, 7(Juliodiciembre), 1-32.

Díaz Barriga, A. (1996). Los programas de evaluación (estímulos al rendimiento académico) en la comunidad de investigadores. Un estudio en la UNAM. Revisa Mexicana de Investigación Educativa, 1(2), 408-423.

Díaz Barriga, A. (1997). Currículum, evaluación y planeación educativa. Ciudad de México: COMIE, CESU, ENEP Iztacala-UNAM, Tomo I.

Díaz Barriga, A., Barrón, C. y Díaz Barriga, F. (2009). Los programas de evaluación en la educación superior mexicana. En H. Aboites et al. (Coords.), Evaluar para la homogeneidad. La experiencia mexicana en la evaluación de la educación superior (pp. 63-85). Ciudad de México: Universidad Autónoma de Sinaloa y Plaza y Valdés.

Díaz Barriga, F. y Díaz Barriga, A. (2008). El impacto institucional de los programas de evaluación de los académicos en la educación superior. En A. Díaz Barriga (Coord.), Impacto de la evaluación en la educación superior mexicana. Un estudio en las universidades públicas estatales (pp.165-221). Ciudad de México: UNAM, IISUE, ANUIES y Plaza y Valdés Editores.

Ducoing, P. y Landesmann, M. (1996). Sujetos de la educación y formación docente. La investigación educativa en los ochenta. Perspectivas para los noventa. Cuidad de México: COMIE.

Estévez, E. (2009). El doctorado no quita lo tarado. Pensamiento de académicos y cultura institucional en la Universidad de Sonora: significados de una política pública para mejorar la educación superior en México. Ciudad de México: ANUIES.

Estévez, E. y Martínez, J. M. (2011). El peso de la docencia y la investigación desde la visión de los académicos de una universidad pública mexicana. El caso de la Universidad de Sonora. Archivos Analíticos de Políticas Educativas, 19(12), 1-26.

Estrada, I. y Guillermo, M. C. (2005). El programa de estímulos al desempeño del personal docente de la UADY: características de los académicos participantes y su implementación de 2001 a 2003. Educación y Ciencia, Nueva Época, 9(32), 23-39. 
Furlán, A. (1998). La evaluación de los académicos. En M. Landesmann, J. M. Mancilla y M. Rueda (Coords.), La evaluación de los académicos: reflexiones e investigaciones (pp. 7-16). Ciudad de México: ENEP Iztacala-UNAM.

Gaceta UNAM. (1993). Anuncia el Rector nuevos estímulos para académicos. UNAM, 1993, 1-5.

Gaceta UNAM. (2001). Programa de Primas al Desempeño del Personal Académico de Tiempo Completo (PRIDE). UNAM, 2001, 18-20.

Galaz, J. (2003). La satisfacción laboral de los académicos mexicanos en una universidad pública estatal: la realidad institucional bajo la lente del profesorado. Ciudad de México: ANUIES.

Galaz, J., Gil, M., Padilla, L. Sevilla, J. J., Arcos, J y Martínez, J. (2012). La profesión académica en México: cambios, continuidades y retos, 1922-2008. En J. Galaz (Coord.), La reconfiguración de la profesión académica (pp. 103-128). Ciudad de México: Universidad Autónoma de Sinaloa y Universidad Autónoma de Baja California.

García, S. (1998). Evaluación académica: recuento curricular e itinerarios biográficos. En M. Landesmann, J. M. Mancilla y M. Rueda (Coords.), La evaluación de los académicos: reflexiones e investigaciones (pp. 28-35). Ciudad de México: ENEP Iztacala-UNAM.

García, S., Grediaga, R. y Landesmann, M. (2003). Los académicos en México. Hacia la constitución de un campo de conocimiento 1993-2002. En P. Ducoing (Ed.), Sujetos, actores y procesos de formación, Tomo I de la colección "La investigación Educativa en México (1992-2002)” (pp.113-268). Ciudad de México: COMIE-SESIC-CESU.

Grediaga, R. (2006). Las políticas hacia los académicos en las últimas décadas. Cambios en la regulación de las trayectorias y el sistema de reconocimiento y recompensas de la profesión académica en México. CPU-e Revista de Investigación Educativa, 2(enero-junio), 172 .

Grediaga, R. y López, R. (2011). Aportaciones a la agenda de investigación sobre educación 2010-2020. Ciudad de México: UAM Azcapotzalco.

Ibarra, E. (2007). De la evaluación del trabajo académico al reconocimiento de las trayectorias: Por un nuevo modelo de la carrera académica. En J. Gandarilla (Coord.), Restructuración de la universidad y del conocimiento (pp. 135-149). Ciudad de México: Centro de Investigaciones Interdisciplinarias en Ciencias y Humanidades-UNAM.

Ibarra, E. y Porter, L. (2007). Costos de la evaluación en escenarios de continuidad: Lecciones mexicanas sobre las disputas con los mercaderes del templo del saber. Espacio Abierto Cuaderno Venezolano de Sociología, 16(1), 61-88.

Kent, R. (1986). Las vicisitudes de una azarosa profesionalización. Crítica, Revista de la Universidad Autónoma de Puebla, 28, 5-19.

Kent, R. (1992/1993). La política de evaluación en la educación superior mexicana. Nóesis, 9-10, 39-53.

Landesmann, M., García, S. y Gil, A. (1996). Los académicos en México: un mapa inicial del área de conocimiento. En P. Ducoing y M. Landesmann (Coords.), Sujetos de la educación y formación docente. La investigación educativa en los ochenta. Perspectivas para los noventa (pp. 67-92). Ciudad de México: COMIE.

Malo, S. y Rojo, L. (1996). Estímulos para la productividad científica y las actividades docentes y artísticas en México. El sistema Nacional de Investigadores. Interciencia, 21(2), 71-79. 
Ordorika, I. (2004). El mercado en la academia. En I. Ordorika (Coord.), La academia en jaque. Perspectivas políticas sobre la evaluación de la educación superior en México (pp. 35-74). Ciudad México: CRIM, UNAM.

Ordorika, I. y Navarro, M. A. (2006). La investigación académica y las políticas públicas en la educación superior; el caso mexicano de pagos por méritos. Revista del Centro de Estudios y Documentos sobre la Educación Superior Puertorriqueña (CEDESP), 1, 53-72.

Pallán, C. (1994, julio). El Programa Nacional de Superación Académica., Ponencia en la $V$ Reunión Ordinaria del Consejo de Universidades Públicas e Instituciones Afines de la ANUIES. Ciudad de Oaxaca, México.

Patron, R. y Cisneros-Cohernour, E. (2014). Los sistemas de estímulos académicos y la evaluación de la docencia: experiencia de dos universidades. Revista Iberoamericana de Evaluación Educativa, 7(2), 85-95.

PEPASIG. (2017). Programa de Estímulos a la Productividad y al Rendimiento del Personal Académico de Asignatura. Recuperado de http://dgapa.unam.mx/index.php/estimulos/pepasig

PRIDE. (2016). Dirección general de asuntos del personal académico programa de primas al desempeño del personal académico de tiempo completo (PRIDE). Recuperado de http://www.iztacala.unam.mx/spcc/pdf/PRIDE2017_convocatoria.pdf

PROMEP. (1996). Programa de Mejoramiento del Profesorado de las Instituciones de Educación Superior. ANUIES. Recuperado de http://resu.anuies.mx/archives/revistas/Revista101_S3A4ES.pdf

Ramírez, M. D. y Padilla, L. (2008). El síndrome del desgaste profesional en académicos. Ciudad de México: Universidad Autónoma de Aguascalientes.

Remedi, E. (2006). Calidad y sufrimiento en la búsqueda desbocada de la excelencia. En M. Landesmann (Coord.), Instituciones Educativas. Instituyendo disciplinas e identidades (pp. 6188). Ciudad de México: Casa Juan Pablos.

Rodríguez-Gómez, R. (1998). Expansión del sistema educativo superior en México 1970- 1995. En M. Fresán Orozco (Ed.), Tres décadas de políticas del Estado en la educación superior (pp. 167-205). Ciudad de México: ANUIES.

Rubio, J. (2006). Financiamiento. En J. Rubio (Coord.), La política educativa y la Educación Superior en México. 1995-2006: Un balance (pp. 186-207). Ciudad de México: SEP-Fondo de Cultura Económica.

Rueda, M. (1998). La evaluación de los académicos en la Universidad vía los programas de compensación salarial. En M. Landesmann, J. M. Mancilla y M. Rueda (Coords.), La evaluación de los académicos: reflexiones e investigaciones (pp. 49-58). Ciudad de México: ENEP Iztacala-UNAM.

Rueda, M. (Coord.). (2011). ¿Evaluar para controlar o para mejorar?: Valoración del desempeño docente en las universidades. Ciudad de México: Bonilla Artiga Editores.

Rueda, M. y Torquemada, A. (2004). Algunas consideraciones para el diseño de un sistema de evaluación de la docencia en la universidad. En M. Rueda (Coord.), ¿̇Es posible evaluar la docencia en la universidad? Experiencias en México, Canadá, Francia, España y Brasil (pp. 2936). Ciudad de México: ANUIES-Universidad Autónoma Benito Juárez de Oaxaca.

Ruiz de la Torre, G. y Rueda, D. X. (s/f). Los procesos de evaluación docente en las universidades autónomas de México. Recuperado de http://www.saece.org.ar/docs/congreso5/trab064.pdf 
Saucedo, C., Guzmán, C. Sandoval, E. y Galaz, J. (Coords.). (2013). Estudiantes, maestros y académicos en la investigación educativa. Tendencias, aportes y debates 2002-2011. Colección Estados del Conocimiento. Ciudad de México: ANUIES-COMIE.

SEP. (2001). Programa Nacional de Educación 2001- 2006. Recuperado de http://www.lie.upn.mx/docs/Especializacion/Planeacion/Metod_SEPE.pdf

SEP. (2011). Programa Integral de Fortalecoimiento Institucional (PIFI). Recuperado de http://pifi.sep.gob.mx/resultados/docs/Impacto_PIFI_2002_2011.pdf

SEP. (2015a). Programa de Estímulos al Desempeño del Personal Docente. Recuperado de http://dsa.sep.gob.mx/informacionadicional.html

SEP. (2015b). Diario Oficial, Decimoprimera Sección. Recuperado de https://www.gob.mx/cms/uploads/attachment/file/46114/docencia.pdf

SHCP. (2002). Lineamientos Generales para la operación del Programa de Estímulos al Desempeño del Personal Docente de Educación Media Superior y Superior. Recuperado de https://www.uv.mx/dgdaie/files/2012/11/SHCPLineamientosEstimulos2002.pdf

Silva, C. (2007). Evaluación y burocracia: medir igual a los diferentes. Revista de la Educación Superior, XXXVI, 3(143), 7-24.

\section{Breve CV del autor}

\section{Patricia Covarrubias Papahiu}

Licenciada por la Facultad de Psicología y doctora en Pedagogía por la Facultad de Filosofía y Letras, UNAM. Profesora titular TC, carrera de Psicología, FES Iztacala, UNAM. Miembro del SNI. Autora y responsable académica del diplomado "Psicología del Trabajo" en la FES Iztacala. Impatición de cursos a nivel licenciatura, maestría y doctorado, en las áreas de Psicología educativa, Psicología organizacional y Metodología de la investigación educativa. Participación y coordinación de proyectos de investigación sobre currículo, enseñanza de las ciencias, procesos de enseñanza y aprendizaje, y construcción del conocimiento en el aula universitaria. Participación en más de 80 congresos nacionales e internacionales de Educación y Psicología, y publicación de resultados en revistas especializadas, en libros y capítulos de libros. Email: papahiu@unam.mx 